\begin{tabular}{|c|c|c|}
\hline INESEG & $\begin{array}{l}\text { INTERNATIONAL } \\
\text { ENGINEERING, } \\
\text { SCIENCE AND } \\
\text { EDUCATION } \\
\text { GROUP }\end{array}$ & $\begin{array}{l}\text { Middle East Journal of Science } \\
\text { (2017) 3(2): } 115-128 \\
\text { Published online December 25, } 2017 \quad \text { (http://dergipark.gov.tr/mejs) } \\
\text { doi: } 10.23884 / \text { mejs.2017.3.2.06 } \\
\text { ISSN: } 2536-5312 \\
\text { Received: September 27, } 2017 \quad \text { Accepted: November 08, } 2017\end{array}$ \\
\hline
\end{tabular}

\title{
SOME SHRUB AND TREE TAXA IN THE GRASSLAND-PASTURE AND NATURAL VEGETATION OF TURKEY Mehmet BASBAG ${ }^{*}$, Erdal CACAN ${ }^{2}$, Mehmet Salih SAYAR ${ }^{3}$, Halil KARAN ${ }^{4}$
}

${ }^{1}$ Dicle University, Faculty of Agriculture, Department of Field Crops, Diyarbakir, Turkey ${ }^{2}$ Bingol University, Vocational School of Genc, Department of Crop and Animal Production, Bingol, Turkey

${ }^{3}$ Dicle University, Vocational School of Bismil, Department of Crop and Animal Production, Diyarbakır, Turkey

${ }^{4}$ Firat University, Vocational School of Sivrice, Department of Crop and Animal Production, Elazig, Turkey

*Corresponding author; mbasbag@ dicle.edu.tr

\begin{abstract}
Some shrub and tree taxa identified by different researchers in the meadow-pasture and natural vegetation of Turkey have been summarized in paper. Total 108 genus and 227 taxa were identified from 49 plant families. Although the vast majority of these taxa (165 taxa-72.7\%) had shrub formation and the numbers of taxa in tree formation was only 27 taxa (11.9\%). The other 35 plant taxa (15.4\%) had a transitional formation between shrub and tree formations. When plants were ordered in terms of family, genus and taxa; Rosaceae was the first number family in 14 genus and 38 taxa (16.7\%), Leguminosae in 12 genus and 35 taxa (15.9\%), and Labiatae family in 11 genus and 25 taxa (11.0\%) followed it. Even though the most taxa possessing families for shrub formation were Leguminosae (36 taxa), Labiatae (25 taxa) and Rosacea (21 taxa), for tree formation the ranking was as Fagaceae (5 taxa), Anacardiaceae (3 taxa), Ericaceae, Cupressaceae and Moraceae (2 taxa). Fagaceae (5 taxa), Anacardiaceae (3 taxa), Ericaceae, Cupressaceae ve Moraceae (2 taxa) families respectively had the most taxa belonging to transitional formation between shrub and tree formations. The results showed that the Kermes oak (Quercus coccifera) (in 11 studies), Black shrub (Paliurus spina-christi) (in 9 studies) and Murt shrub (Myrtus communis) (in 7 studies) were the most cited and frequently encountered shrub taxa by researchers in Turkey. Additionally, among the taxa were identified seven taxa as "endemic" and five taxa as "rare" plants.
\end{abstract}

Key words: Grassland, pasture, shrub, tree, taxa 


\section{Introduction}

In Turkey, the grassland-pasture area is 14.611 .920 hectare (ha) and this accounts for $18.8 \%$ of the country's land [37]. Total size of pasture lands in-forests, side forests and top forests in Turkey are 3.450 .736 ha, which accounts for $21 \%$ of total pasture size of the country [1]. There are about 8.3 million hectares of shrubs area in Turkey. Most of the shrubs consist of the maquis, which is natural vegetation of mediterranean climate. With a legal arrangement, these shrub areas were included in the forest area in 1969 [3]. Generally, due to over, untimely and non-uniform grazings, the amount of quality forage plant species are reduced and even disappeared in rangelands of Turkey [26]. Instead of the quality species, the rangelands are filled with other species, weeds and shrubs whose feed quality is low. Shrubs and trees are commonly seen in meadow and pasture lands that close to forests. Shrubs and trees in grassland-pasture areas are generally accepted as weed. This is due to the following reasons. They reduce the yield and quality of the forages, and they prevent comfortably animal grazing on rangelands [27]. Accordingly, the struggle with them is one of the important topics of pasture improvement programs. The pasture law numbered 4342 comes into force in 1998 in Turkey, pasture breeding studies started to gain speed in the Ministry of Food, Agriculture and Livestock, in Turkey [27]. In the control management with shrubs and tree taxa in the improvement of grassland-pasture, generally mechanical control, chemical control [11, 13, 15, 19, 22, 23 and 39] and biological control methods are used. Furthermore, usually goats are used in the method of biological control [40]. However, some shrubs and tree taxa in the grassland-pasture areas are the source of feed for domestic animals such as goats and sheep. They also contribute to the conservation of biodiversity by creating areas of nutrition and shelter for wild animals.

According to Gokkus et al. (2013), there are a great deal amount dry matter productions from herbaceous plants in the maquis areas. Especially they emphasized that amount of the forage varied depending on the frequency of the shrubs in the area [21]. The most of the sloping lands of in the Cukurova region are widely covered with maquis-shrub vegetation. The altitude of these lands extends up to 500-600 m higher than sea level. These shrubs generally are grazed with sheep and goat herds in winter period. The commonly found shrub species in these maqius are Catycotome infesta, Cistus satviaefolius, Genista sp., Quercus coccifera etc... [36]. Shrubs and small tree taxa are widely encountered in Mediterranean and Aegean Sea coasts up to $500 \mathrm{~m}$ height from sea level. Among the species the widely ones are wild olive (Olea europea L.), arbutas (Arbutus unedo L.), common myrtle (Myrtus communis L.), phllyrea, gum tree (Pistacia lenticus L.), Laden (Cistus erectus L.), Kermes oak (Quercus coccifera L.) and holy oak (Quercus ilex L.) [10]. In Kuredagi pasture land, a forest inside rangeland, rate of Thymus sipyleus species in botanical composition was 0.90\% [1]. In Araplar village pastureland, Turkoglu district, Kahramanmaras the rate of Paliurus spina-christi was 1.15\% [38].

Babalık and Fakir (2007) investigated the effects goats grazing on leaf morphology of some shrub species (Quercus coccifera, Crataegus orientalis var. orientalis and Cotoneaster nummularia) in Kozagac1 plateau, Davraz Mountain, Isparta province. They were reported that the fragrant Juniper (Juniperus foetidissima) and the Greek Juniper (Juniperus excelsa) taxa converted their formation from tree formation into shrub formation due to intensive grazing [9]. In Kilis province, Quercus coccifera, 
Ziziphora clinopodioides and Thymus sipyleus covered rate of $0.81-2.09 \%, 0.43-1.34 \%$ and $3.21 \%$, respectively, in botanical composition of some pasture lands [31]. Weed species amount in rangelands of transitional zone of Cukurova region was \% 58.7. Among the weed species, Murt shrubs (Myrtus communis) ratio was in 8.1\% [13]. Serbus eria shrub taxa were covered 2.5\% in Samsun province [41]. Rubus sanctus shrub species was covered $1.9 \%$ of flat rangelands in Kırlkhan district, Hatay province [14].

Gokkus et al. (2009) determined protein ratio of green biomass in Phllyrea, Quercus coccifera shrub taxa 5.56-7.61\% to 5.63-7.25\%, respectively in Canakkale province, Turkey [20]. In Isparta province, 1 hectare shrub area, covered with Quercus coccifera, is sufficient for 4 goats during a grazing period [35].

Alaturk et al. (2014) as result of their study conducted in Canakkale with 9 different shrub species, Phllyrea, Kermes oak, Cyprus oak, Gorse, Genista, Sea grape, Pseudoacacia, Goat bilberry and Prickly juniper, they reported that some important traits were ranged as followings; crude protein content 5.3416.31\%; crude oil content 4.46-7.57\%; tannin content 0.11-2.18\%; NDF ratio 35.22-53.87\%; ADF ratio $23.77-44.15 \%$ and ADL ratio 8.54-16.98\% [2].

Çetiner et al. (2015) reported that pre-improvement and management studies, 20-50\% of the rangeland were covered with shrubs in Gerlengec village, Biga district, Canakkale province, Turkey [12].

Astragalus microcephalus and Astragalus macrocephalus species were found in high parts of Karacadag Mountains, located in the Southeast Anatolia. Moreover, these Astragalus species had shrub form and they were main feed sources for goat and sheep herds in the zone [27]. Alhagi pseudalhagi, shrub taxa, covers in 0.6-0.8\% in the botanical composition of the pasture lands of Bismil district, Diyarbakır province [30]. The Kermes oak (Quercus coccifera), having a shrub or small tree form and predominant species, was used in feeding of goats due to its low input by people of Aegean and Mediterranean regions of Turkey [39]. The most economical control can be done against weed species in the pasture lands of Turkey with using goats in grazing. In this context, the most problematic species are Centaurea, Cirsium and Rumex having herbaceous formation, and Rubus, Rosa and Genista having shrub formation [40].

In this review study some shrub and tree taxa found in the grassland-pasture lands and natural vegetation of Turkey were examined.

\section{Plant Growth Forms and Status of the Taxa}

In this study, some species of shrub and tree taxa which have been determined by different researchers in the grassland-pasture areas and natural vegetation of Turkey have been sumarized. In the study, Latin, Turkish, English names, growth habitus and taxa for each family are given in the Table 1 cited by previous studies $[1,4,5,6,7,8,9,10,11,12,13,14,15,16,17,18,22,23,24,25,27,28,29$, $30,31,33,34,36,37,39,40,41$ and 42]. Total 108 genus and 227 taxa belonging to 49 families in the grassland-pasture and natural vegetation of Turkey were determined (Table 1).

Detailed groupings were made by growth patterns of plant taxa (Table 1). The numbers of plant taxa in shrub forming were 107, and this indicated as the shrub (107). The other growth forms and taxa numbers were as followings; shrubby (17), half-shrubby (11), shrub or shrubby (6), half-shrub (4), scrub 
shrub (2), shrub, half-shrub (2), shrub, dwarf-shrub (1), shrub, small shrub (1), shrub, aquatic shrub (1), shrub, half-shrubby (1), shrubby or half-shrubby (1), shrubby, shrub or woody-herb (1), small shrubby (1), small shrubby, herb or shrub (1), herb or half-shrub (1), herb or shrub (1), herb or shrubby (1), herbaceous-shrubby, half-shrub (1), wrapping shrub (1), creeping shrub (1), half-shrubby, shrubby (1), half-shrubby, herb or half-shrub (1) as "Shrub"; tree (17), shrub-tree (7), small tree (2), small shrub-tree (1) as "Trees" and shrub or tree (14), shrub or small tree-shrub (6), shrub or tree-shrub (3), shrub or tree (2), shrub or small tree (2), tree or shrub (1), tree or tall shrub (1), small shrub or shrub (1), shrub or scrub tree (1), shrub or small shrub-tree (1), shrub or rarely tree (1), shrub or rarely small tree (1), shrubby or woody (1).

The plant taxa were mainly grouped for three plant formations (Table 2). Plant taxa grouped; 165 taxa $(72.7 \%)$ in shrub formation, 27 taxa (11.9\%) in tree formation and 35 taxa (15.4\%) in a transitional formation between shrub and tree formations.

Leguminosae (Fabaceae) family is the first number family with 36 taxa. It was followed by Labiatae (Lamiaceae) and Rosacea families with 25 and 21 shrub taxa, respectively. Even though 7 shurb taxa found in Ericaceae and Rhamnaceae families, 6 taxa found with shrub formation in Chenopodiaceae and Cupressaceae families. Rosaceae family ( 8 taxa) was the most numbers tree formation found taxa. It was followed by Fagaceae, Salicaceae, Cannabaceae families with 2 taxa, and Cupressaceae, Anacardiaceae, Oleaceae, Cornaceae, Pinaceae ve Ulmaceae with 1 taxa having tree formation and transitional formation between shrub and tree formations. The most taxa found in Fagaceae family with 5 taxa, it was followed by Anacardiaceae family with 3 taxa and Ericaceae, Cupressaceae and Moraceae faimilies with 2 taxa (Table 2). Furthermore, the status of the 10 families in terms of numbers of taxa belonging to 3 formations, shrub, tree and shrub-tree is indicated in Figure 1.

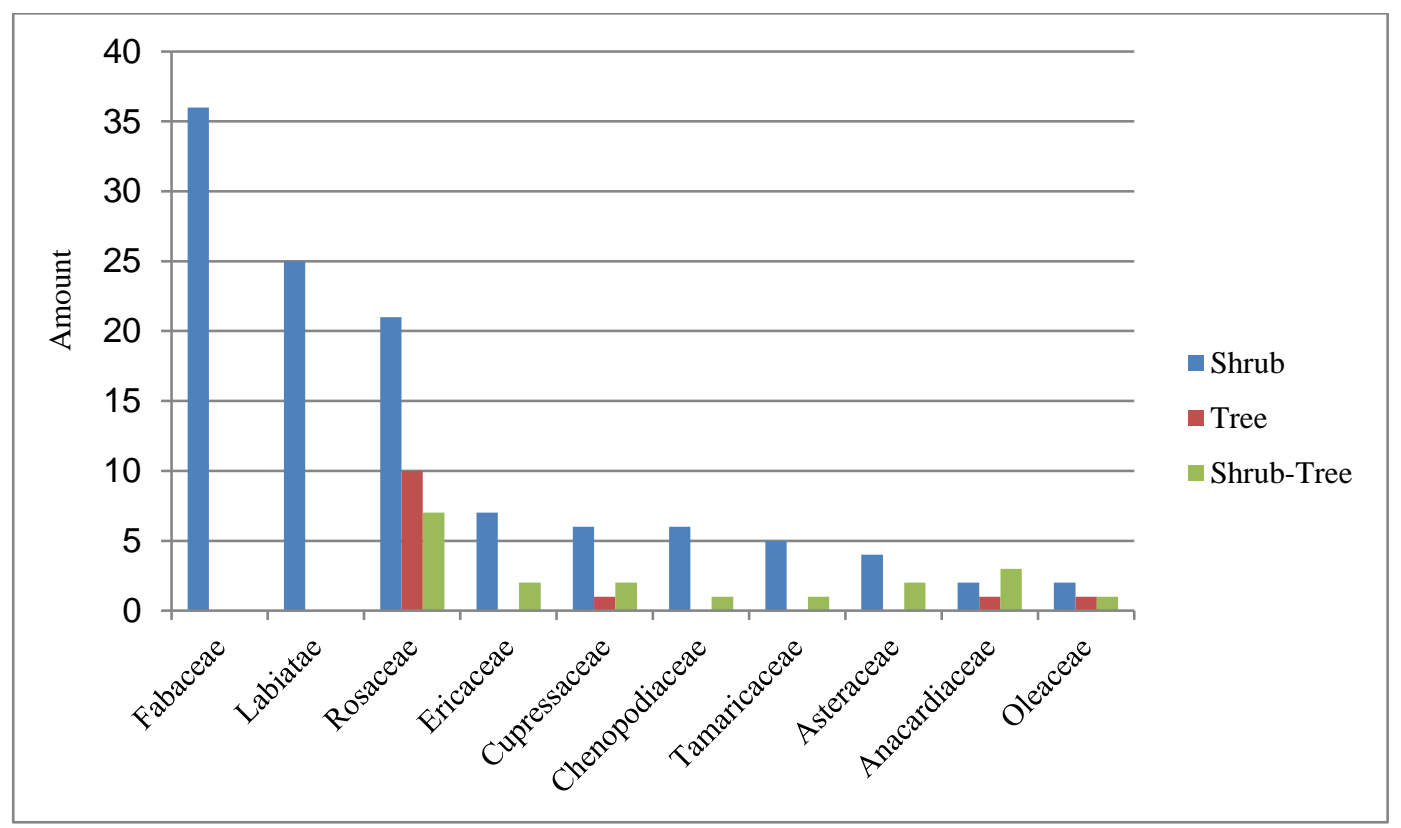

Figure 1. Numbers of plant taxa for shrub, tree and shrub-tree formations in the the most 10 common plant Families 


\section{Important Shrubs Taxa and Families in Turkey}

Among 49 plant family, Rosaceae family was found as possessing the most numbers in 14 genus (13.0\%.) and 38 taxa (16.7\%). Rosaceae family was followed by Leguminosae (Fabaceae) in 12 genus $(11.1 \%)$ and 36 taxa (15.6\%), Labiatae (Lamiaceae) in 11 genus (10.2\%) and 25 taxa (11.0\%), Chenopodiaceae in 7 genus (6.48\%) and 7 taxa (3.08\%) and Ericaceae in 4 genus (3.70\%) and 9 taxa (\%3.96) (Table 2). In addition, the first 10 family, possessing the most number genus and taxa, were given in the Figure 2.

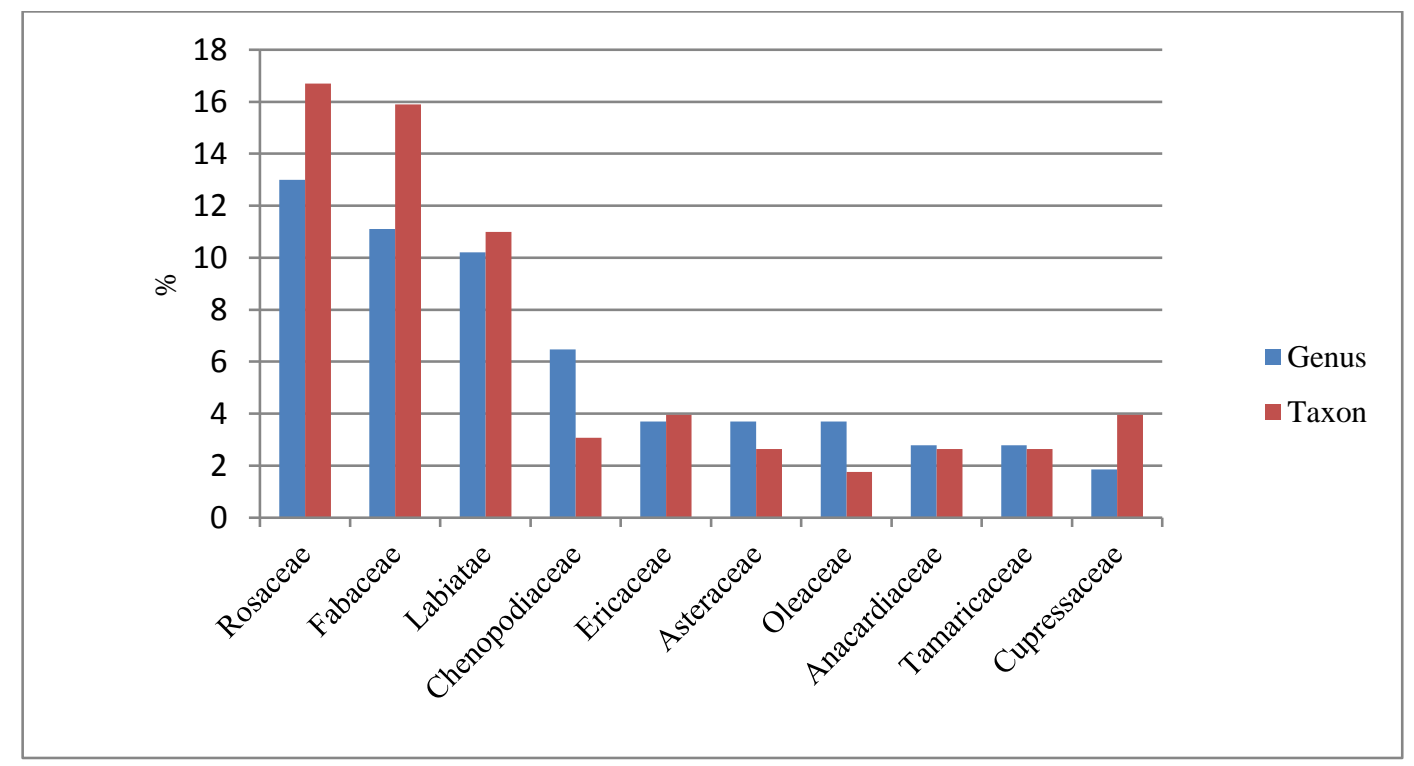

Figure 2. Numbers (\%) of taxa and genus in the the most 10 common plant families, including shrub and tree forms plants

Among the taxa having shrub formation, the most found taxa is Kermes oak (Quercus coccifera) in Turkey. It was cited in 11 studies. It was followed by Black shrub (Paliurus spina-christi) (cited in 9 studies), Murt shrub (Myrtus communis) (cited in 7 studies), Thornburnet (Sarcopoterium spinosum), Blackberry (Rubus sanctus), Small-headed astragalus (Astragalus microcephalus) (cited in 6 studies), Hairy germander (Teucrium polium), Chaste (Vitex agnus-castus) and Common camel thorn (Alhagi pseudalhagi) (cited in 5 studies).

Also, one of the most remarkable points of this work was that 7 shrub taxa were found as endemic (END LR 1c). The endemic taxa were Astragalus acicularis, Astragalus baibutensis, Astragalus condensatus, Astragalus cymbbostegius, Genista aucheri, Rhamnus hirtellus and Veronica multifida. On the other hand, 5 shrub taxa were found as non-endemic rare plants (NB VU) [16]. These nonendemic rare plants taxa were Alhagi mannifera, Galium incanum, Globularia cordifolia, Salvia fruticosa, Thymus transcaucasicus (Table 1) [16]. 


\section{Conclusions}

Totally, 108 genus and 227 taxa were identified from 49 plant families in the scope of this study. It was also revealed that shrub, tree and transitional formation between shrub and tree formations had respectively $165(72.7 \%)$ taxa, 27 taxa (11.9\%) and 35 plant taxa (15.4\%). Among all of the shrub taxa, the Kermes oak (Quercus coccifera), Black shrub (Paliurus spina-christi), Murt shrub (Myrtus communis), Thornburnet (Sarcopoterium spinosum), Blackberry (Rubus sanctus) and Small-headed astragalus (Astragalus microcephalus) have been the most reported taxa by the researchers in Turkey. In conclusion, long as uncontrolled and overgrazing continues in the grassland and pasture lands, quality forage crop species will disappear from these areas. The weeds and shrub taxa will continue to take the place of the quality forage plants.

Table 1. Some of the shrub and tree taxa found in the grassland-pasture and natural areas of Turkey $(1,4,5,6,7,8,9,10,11$, $12,13,14,15,16,17,18,22,23,24,25,27,28,29,30,31,33,34,36,37,39,40,41$ and 42 )

\begin{tabular}{|c|c|c|c|c|}
\hline Species Name & Life Forms & Turkish Name & English Name & Family \\
\hline Acantholimon acerosum (Willd.) Boiss. & Shrubby & Sert kar dikeni, Çobanyastığı & & Plumbaginaceae \\
\hline $\begin{array}{l}\text { Acantholimon acerosum (Willd.) Boiss. var. } \\
\text { acerosum }\end{array}$ & Shrubby & Pisik geveni & & Plumbaginaceae \\
\hline $\begin{array}{l}\text { Acantholimon ulicinum (Willd. Ex Schultes) } \\
\text { Boiss. }\end{array}$ & Shrub, Small shrub & $\begin{array}{l}\text { Pisik geveni, Çobanyastığı, Kurak } \\
\text { kar dikeni }\end{array}$ & Prickly thrift & Plumbaginaceae \\
\hline Acantholimon venustum Boiss. & Shrubby & Kar dikeni & Agrimony, Churchsteeples & Plumbaginaceae \\
\hline Acer monspessulanum $\mathrm{L}$. & Shrub or Scrub tree & Fransız akçaağacı & Montpellier maple & Aceraceae \\
\hline Alhagi mannifera Desv. (NB VU) & Shrub or Shrubby & Deve dikeni & Camelthorn & $\begin{array}{c}\text { Leguminosae } \\
(\text { Fabaceae })\end{array}$ \\
\hline Alhagi pseudalhagi (M.Bieb.) Desv. & Shrub or Shrubby & Adi deve dikeni, Kara yandık & Camelthorn & Leguminosae \\
\hline Amygdalus communis $\mathrm{L}$. & Shrub or Tree & Acıbadem ağacı & Almon Tree & Rosaceae \\
\hline Amygdalus orientalis Miller & Shrub & Keçi bademi & Goad almond & Rosaceae \\
\hline Arbutus andrachne $\mathrm{L}$. & Shrub & Sandal ağacı, Hartlap & Strawberry tree & Ericaceae \\
\hline Arbutus unedo L. & Shrub & Dağ çileği, Kocayemiş & Strawberry tree & Ericaceae \\
\hline Artemisia absinthium $\mathrm{L}$. & Half shrubby & $\begin{array}{l}\text { Pelin otu, Ac1 yavşan, Ak pelin, } \\
\text { Büyük yavşan otu }\end{array}$ & Absinthium & $\begin{array}{c}\text { Compositae } \\
\text { (Asteraceae) }\end{array}$ \\
\hline Artemisia santonicum $\mathrm{L}$. & $\begin{array}{l}\text { Half shrubby, } \\
\text { Shrubby }\end{array}$ & $\begin{array}{l}\text { Yavşan otu, Deniz yavşana, Deniz } \\
\text { pelini, Kokulu yavşan }\end{array}$ & Sagebrush & Compositae \\
\hline Asparagus acutifolius $\mathrm{L}$. & Shrubby & $\begin{array}{l}\text { Çıtır, Tilkişen, Acı ot, Dikenli acı } \\
\text { ot, Kırgın otu, Yabani kuşkonmaz, }\end{array}$ & & Liliaceae \\
\hline Astragalus acicularis Bunge (END LR lc) & Shrub & & & Leguminosae \\
\hline Astragalus andrachneifolius Fenzl & Shrub & Geven & Astragale & Leguminosae \\
\hline Astragalus aureus Willd. & Shrub & Sarıçiçekli geven, Altın geveni & Astragale & Leguminosae \\
\hline Astragalus baibutensis Bunge (END LR lc) & Shrub & Geven & Milk-vetch & Leguminosae \\
\hline Astragalus condensatus Ledeb. (END LR lc) & Shrub & Yastıklı geven & & Leguminosae \\
\hline Astragalus cymbostegius Bun. (END LR lc) & Shrub & Geven & Astragale & Leguminosae \\
\hline Astragalus gumnifer Lab. & Shrub or Shrubby & $\begin{array}{l}\text { Geven, Ak geven, Sakız geveni, } \\
\text { Püs geveni, Zamk geveni }\end{array}$ & Gum tragacanth & Leguminosae \\
\hline Astragalus lagurus Willd. & Shrub & Tüy başlı geven & Astragale & Leguminosae \\
\hline Astragalus macrocephalus Willd. & Shrub or Shrubby & $\begin{array}{l}\text { Küçükbaşl geven, Boz geven, } \\
\text { Kara geven }\end{array}$ & Astragale & Leguminosae \\
\hline Astragalus microcephalus Willd. & Shrub or Shrubby & $\begin{array}{l}\text { Küçükbaşl geven, Boz geven, } \\
\text { Kara geven }\end{array}$ & Astragale & Leguminosae \\
\hline Astragalus ponticus Pallas & Half shrubby & Karadeniz geveni, Laz geveni & Pontic milk-vetch, Astragale & Leguminosae \\
\hline Astragalus prusianus Boiss. & Shrub & & & Leguminosae \\
\hline Astragalus trojanus Stev. & Shrub, Half shrubby & Truva geveni & & Leguminosae \\
\hline Astragalus wiedemannianus Fischer & Shrub & & & Leguminosae \\
\hline Atriplex halimus $\mathrm{L}$. & Shrub & Yabani pazı, Parlak karapazı & Orache & Chenopodiaceae \\
\hline Berberis crataegina DC. & Shrub & $\begin{array}{l}\text { Adi hanımtuzluğu, Siyah meyveli } \\
\text { hanımtuzluğu, Karamuk }\end{array}$ & Barberry, Cretan barberry & Berberidaceae \\
\hline Berberis cretica $\mathrm{L}$. & Shrub & Siyah meyveli hanımtuzluğu & Barberry & Berberidaceae \\
\hline Berberis integerrima Bunge & Shrub & Hanımtuzluğu, Karamuk & \begin{tabular}{|l|} 
Barberry \\
\end{tabular} & Berberidaceae \\
\hline Berberis vulgaris $\mathrm{L}$. & Shrub & $\begin{array}{l}\text { Dağ kadıntuzluğu, Anberparis, } \\
\text { Karamuk, Zibike }\end{array}$ & $\begin{array}{l}\text { Common barberry, European } \\
\text { barberry }\end{array}$ & Berberidaceae \\
\hline Buxus sempervirens $\mathrm{L}$. & Shrub & Şimşir & Common box, European box & Buxaceae \\
\hline Calicotome infesta (C. Presl) Guss. & Shrub & & & Leguminosae \\
\hline Calicotome villosa (Poiret) Link & Shrub & & & Leguminosae \\
\hline Camphorosma monspeliaca $\mathrm{L}$. & Shrub & & & Chenopodiaceae \\
\hline Capparis ovata Desf. & Half shrub & Kapari, Kebere, Gebele & Caper bush, Common caper & Capparaceae \\
\hline Capparis ovata Desf. var. canescens & Shrub, Half shrub & Kebere, Gebre otu, Kapari & Caper bush & Capparaceae \\
\hline Capparis ovata Desf. var. herbaceae & Shrub, Half shrub & Kebere, Kapari & Caper bush & Capparaceae \\
\hline
\end{tabular}




\begin{tabular}{|c|c|c|c|c|}
\hline Capparis spinosa $\mathrm{L}$. & Shrub & Kapari, Gebere, Kedi tırnağ & Caper & Capparaceae \\
\hline Capparis spinosa L. var. spinosa & Half shrub & Kebere, Kapari & Caper & Capparaceae \\
\hline Caragana grandiflora (Bieb.) DC. & Shrub & & & Leguminosae \\
\hline Carpinus betulus $\mathrm{L}$. & Tree & Karagürgen, Orsit & European hornbeam & Betulaceae \\
\hline Celtis glabrata Steven ex Planchon & Tree & Çitlenbik, Dardağan & & Cannabaceae \\
\hline Celtis tournefortii Lam. & Tree & Çitlenbik, Dardağan & & Cannabaceae \\
\hline $\begin{array}{l}\text { Cerasus microcarpa (C.A. Meyer) Boiss. } \\
\text { subsp. tortuosa (Boiss. \& Hausskn.) Browicz }\end{array}$ & Small tree & & & Rosaceae \\
\hline Chamaecytisus pygmaeus (Willd.) Rothm. & Shrub & & & Leguminosae \\
\hline Cistus creticus $\mathrm{L}$. & Shrub & Karağan & Cretan rockrose & Cistaceae \\
\hline Cistus erectus $\mathrm{L}$. & Tree & Laden & & Cistaceae \\
\hline Cistus salviifolius $\mathrm{L}$. & Shrub & Adaçayı yapraklı laden & & Cistaceae \\
\hline Clematis orientalis $\mathrm{L}$. & Wrapping shrub & & Clematis & Ranunculaceae \\
\hline Colutea cilicica Boiss. \& Balansa & Shrub & Patlangaç & \begin{tabular}{|l|} 
Blander senna \\
\end{tabular} & Leguminosae \\
\hline Coridothymus capitatus (L.) Rchb. Fil. & Small shrubby & Bal kekiği & & $\begin{array}{c}\text { Labiatae } \\
\text { (Lamiaceae) }\end{array}$ \\
\hline Cornus sanguinea $\mathrm{L}$. & Small shrub-Tree & Kizılcık & Common dogwood & Cornaceae \\
\hline Cotinus coggyria Scop. & Shrub & Boyac1 sumağı, Duman ağac1 & Red smoke bloom, Smoke brush & Anacardiaceae \\
\hline Cotoneaster integerrimus Medik. & Shrub & Dağ muşmulası & Cotoneaster & Rosaceae \\
\hline Cotoneaster nummularia Fisch. \& C.A. Mey. & Shrub or Tree & Dağ muşmulası & & Rosaceae \\
\hline Crataegus aronia (L.) Bosc. ex DC. var. aronia & Small tree & Alıç & Hawthorn & Rosaceae \\
\hline Crataegus curvisepala Lindm. & Shrub or Tree & & & Rosaceae \\
\hline Crataegus microphylla C. Koch & Shrub & & & Rosaceae \\
\hline Crataegus monogyna Jacq. & Small tree & $\begin{array}{l}\text { Alıç, Adi alıç, Akdiken, Adi } \\
\text { akdiken, Yemişen }\end{array}$ & $\begin{array}{l}\text { Oriental Thorn - large } \\
\text { yellow/red haw, Hawthorn }\end{array}$ & Rosaceae \\
\hline Crataegus monogyna Jacq. subsp. monogyna & Small tree & Alıç & Hawthorn & Rosaceae \\
\hline $\begin{array}{l}\text { Crataegus orientalis Pallas ex Bieb. var. } \\
\text { orientalis }\end{array}$ & Small tree & Alıç & Hawthorn & Rosaceae \\
\hline Crataegus orientalis Pallas ex M. Bieb. & Small tree & Alıç & Hawthorn & Rosaceae \\
\hline Crataegus pentagyna Waldst. et Kit.ex Willd. & Shrub or Shrub-tree & & & Rosaceae \\
\hline Cytisopsis dorycniifolia Jaub. et Spach & Shrub & & & Leguminosae \\
\hline Cytisus triflorus L'Hérit. & Shrub & & & Cistaceae \\
\hline Daphne glomerata Lam. & Shrub, Dwarf shrub & Kurtbağ 1, Sırımbağ 1 & \begin{tabular}{|l} 
Daphne \\
\end{tabular} & Thymelaeaceae \\
\hline Daphne gnidioides Jaub. et Spach. & Shrub & Defne, Develik, Havaza & Daphne & Thymelaeaceae \\
\hline Daphne oleoides Shreber & Shrub & Zeytin defnesi, Havadana & Garland-flowered mezereon & Thymelaeaceae \\
\hline Daphne pontica $\mathrm{L}$. & Shrub & Karadeniz yabani defnesi, Kurtbağ 1 & \begin{tabular}{|l|} 
Twin-flowered daphne \\
\end{tabular} & Thymelaeaceae \\
\hline Daphne sericea Vahl. & Shrub & İpeksi defne & & Thymelaeaceae \\
\hline Dittrichia viscosa (L.) Greuter & Shrubby & Andiz otu & & Compositae \\
\hline Dorycnium graecum $($ L.) Ser. & Her or Half shrub & Tüylü yanık üçgülü, Kaplan otu & \begin{tabular}{|l|} 
Canary clover, Trefoil \\
\end{tabular} & Leguminosae \\
\hline Dorycnium hirsutum (L.) Ser. & Herb or Shrub & Ege yanık üçgülü & Gray brom, Hairy canary clover & Leguminosae \\
\hline Dorycnium pentaphyllum Scop. & $\begin{array}{c}\text { Small shrubby, Herb } \\
\text { or Shrub }\end{array}$ & Zehirli yonca, Sarılık yonca & Dorycnium, Socarrillo & Leguminosae \\
\hline Elaeagnus angustifolia $\mathrm{L}$. & $\begin{array}{l}\text { Shrub-Tree or } \\
\text { Shrub }\end{array}$ & İğde & Russian olive & Eleagnaceae \\
\hline Ephedra campylopoda C.A. Meyer & Creeping Shrub & Deniz üzümü & Ephedra & Ephedraceae \\
\hline Ephedra majör Host. & Shrub, Aquatic shrub & Deniz üzümü, Hum & Ma huang & Ephedraceae \\
\hline Erica arborea $\mathrm{L}$ & \begin{tabular}{|c|} 
Shrub \\
\end{tabular} & Süpürge ağ & & Ericaceae \\
\hline Erica manipuliflora Salisb. & Shrub & Funda & Heather & Ericaceae \\
\hline Eriolobus trilobatus (Poiret) Roemer & Small tree & At elması, Ateş dikeni, Ateş yaprağı & Wild apple tree & Rosaceae \\
\hline Ficus carica $\mathrm{L}$. & Shrub or Tree & \begin{tabular}{|l|l} 
Incir \\
\end{tabular} & \begin{tabular}{|l} 
Fig tree \\
\end{tabular} & Moraceae \\
\hline $\begin{array}{l}\text { Ficus carica L. subsp. rupestris (Hausskn.) } \\
\text { Browicz }\end{array}$ & Shrub or Tree & İncir & Fig tree & Moraceae \\
\hline Frankenia hirsuta $\mathrm{L}$. & Herb or Shrubby & Tüylü deniz fundası & Sea heathweed & Frankeniaceae \\
\hline Fraxinus angustifolia Vahl. subsp. angustifolia & Tree & Dişbudak & & Oleaceae \\
\hline Galium incanum Sibth. \& Sm. (NB VU) & $\begin{array}{l}\text { Half shrubby, Herb } \\
\text { or Half shrub }\end{array}$ & Yarı çalı yoğurtotu & Bedstraw & Rubiaceae \\
\hline Genista albida Willd. & Shrub & Katırtırnağ 1 & Soap wort & Leguminosae \\
\hline Genista anatolica Boiss. & Shrub & Boyac1 katırtırnağ1 & & Leguminosae \\
\hline Genista aucheri Boiss. (END LR lc) & Shrub & $\begin{array}{l}\text { Anadolu katırtırnağı, Anadolu } \\
\text { boya çalısı }\end{array}$ & $\begin{array}{l}\text { Petty whin, Anatolian needle } \\
\text { furze }\end{array}$ & Leguminosae \\
\hline Genista carinalis Gris. & Shrub & Katırtırnağ1 & & Leguminosae \\
\hline Genista lydia Boiss. & Shrub & Lidya katırtırnağ1 & Lydian needle furze & Leguminosae \\
\hline Genista sessifolia DC. & Shrub & Katırtırnağı, Boyacı katırtırnağı & Greenweed, Needl Furze & Leguminosae \\
\hline Genista tinctoria $\mathrm{L}$. & Shrub & Boyac1 katırtırnağ1 & & Leguminosae \\
\hline Genista vuralii A. Duran \& H. Dural & Shrub & Katırtırnağ 1 & Greenweed, Needl Furze & Leguminosae \\
\hline Globularia cordifolia $\mathrm{L}$. (NB VU) & Shrub & & & Globulariaceae \\
\hline Halimione portulacoides (L.) Aellen & Shrub & Çorak otu, Tuzlu yalancı palı & \begin{tabular}{|l} 
See purslane \\
\end{tabular} & Chenopodiaceae \\
\hline Halocnemum strobilaceum (Pall.) Bieb. & Scrub shrub & Çuvan, Ac1 ot & & Chenopodiaceae \\
\hline Halostachys belangeriana (Moq.) Botsch. & Shrub or Small tree & & & Chenopodiaceae \\
\hline Hippophae rhamnoides $\mathrm{L}$. & Shrub & Yabani iğde & Seabuckthorn, Sanddorn & Eleagnaceae \\
\hline Hypericum scabrum $\mathrm{L}$. & Shrubby & Kaba kuzu kıran & St. Jonswort & $\begin{array}{c}\text { Guttiferae } \\
\text { (Hypericaceae) }\end{array}$ \\
\hline Ilex colchica Poj. & Shrub or Tree & Çobanpüskülü & English holly & Aquifoliaceae \\
\hline Jasminum fruticans $\mathrm{L}$. & Shrub & $\begin{array}{l}\text { Yabani yasemen, Boruk, } \\
\text { Borumuk, Karaporuk }\end{array}$ & Wild jasmine & Oleaceae \\
\hline Juniperus communis L. & Shrub & Adi ardıç, Bodur ardıç & Common juniper & Cupressaceae \\
\hline Juniperus communis L. subsp. nana Syme & Shrub & Adi ardıç, Bodur ardıç & Common juniper & Cupressaceae \\
\hline Juniperus excelsa M. Bieb. & Shrub & Boylu ardıç & & Cupressaceae \\
\hline Juniperus foetidissima Willd. & Shrub & Kokulu ardıç & & Cupressaceae \\
\hline Juniperus irombinus & Shrub & & & Cupressaceae \\
\hline Juniperus oxycedrus L. & Shrub or Small tree & Katran ardic 1 & Prickly juniper, Prickly cedar & Cupressaceae \\
\hline
\end{tabular}




\begin{tabular}{|c|c|c|c|c|}
\hline Juniperus phoenicia $\mathrm{L}$. & Shrub-Tree & Finike ardıcı & Juniper & Cupressaceae \\
\hline Juniperus sabina $\mathrm{L}$. & Scrub shrub & İtalyan ardıc1, Sabin ardıc1 & Savin, Savine & Cupressaceae \\
\hline Kochia prostrata (L.) Schrad. & $\begin{array}{c}\text { Shrubby or Half } \\
\text { shrubby }\end{array}$ & Süpürge otu, Adi bozkır otu & $\begin{array}{l}\text { Summer cypress, Mock cypres, } \\
\text { fireweed }\end{array}$ & Chenopodiaceae \\
\hline Laurus nobilis L. & Shrub or Tree & $\begin{array}{l}\text { Defne, Har, Nehtel, Tahnal, } \\
\text { Tefrün, Tehnel, Tenel }\end{array}$ & & Lauraceae \\
\hline Linum aroanium Boiss. et Orph. & Half shrub & & & Linaceae \\
\hline Lonicera xylosteum $\mathrm{L}$. & Shrub & Hanımeli & Fly honeysuckle & Caprifoliaceae \\
\hline Malus communis $\mathrm{L}$. & Tree & Elma & Apple & Rosaceae \\
\hline Myricaria germanica (L.) Desv. & Shrub & Alman 1lgını, Yalanc1 1lgın & False tamarisk & Tamaricaceae \\
\hline Myrtus communis $\mathrm{L}$. & Shrub & Mersin, Murt, Murt çalısı & Myrtle & Myrtaceae \\
\hline Nerium oleander $\mathrm{L}$. & Shrub & Zakkum & Oleander & Apocynaceae \\
\hline Nitraria schoberi L. & Shrub & & & Zygophyllaceae \\
\hline Noaea mucronata (Forssk.) Aschers. et Sch. & Shrub & Dikenli ölmez otu & Goosefoots & Chenopodiaceae \\
\hline Oenanthe silaifolia Bieb. & Shrub & Damla otu & $\begin{array}{l}\text { Narrow-leaved water-dropwort, } \\
\text { Sulpherwort }\end{array}$ & Umbelliferae \\
\hline Olea europaea $\mathrm{L}$. & Shrub & Yabani zeytin, Delice & Wild olive & Oleaceae \\
\hline Onobrychis cornuta $(\mathrm{L}$.$) Desv.$ & Shrub or Shrubby & Boynuzlu korunga, Dağ çöveni & & Leguminosae \\
\hline Origanum onites $\mathrm{L}$. & $\begin{array}{c}\text { Herbaceous- } \\
\text { Shrubby, Half shrub }\end{array}$ & $\begin{array}{l}\text { İzmir kekiği, Kaba tüylü } \\
\text { mercanköşs }\end{array}$ & $\begin{array}{l}\text { Cretan oregano, Rhigani, Pot } \\
\text { marjoram }\end{array}$ & Labiatae \\
\hline Origanum sipyleum $\mathrm{L}$. & Half shrub & Bayır Çayı, Güvey otu & & Labiatae \\
\hline Origanum syriacum $\mathrm{L}$. & Shrubby & Dağ kekiği & Syrian oreganu & Labiatae \\
\hline Paliurus spina-christi Miller & Shrub & $\begin{array}{l}\text { Karaçal, Çalı dikeni, Çaltı dikeni, } \\
\text { Çeşmezen, İsa dikeni, Kara çaltı, } \\
\text { Kara diken, Kışla dikeni, Kunar, } \\
\end{array}$ & Jarusalem thorn, Garland thorn & Rhamnaceae \\
\hline Phillyrea latifolia $\mathrm{L}$. & Shrub or Small tree & Kesme, Akçakesme, Akçe kesme & & Oleaceae \\
\hline Phlomis kotschyana Hub. -Mor. & Shrubby & Kudüs adaçayı & Jarusalem sage & Labiatae \\
\hline $\begin{array}{l}\text { Pinus nigra Arn. subsp. pallasiana (Lamb.) } \\
\text { Holmboe }\end{array}$ & Tree & Anadolu karaçamı & & Pinaceae \\
\hline Pinus nigra J. F. Arnold & Tree & Çam ağacı & & Pinaceae \\
\hline Pistacia atlantica Desv. & Tree or Shrub & Atlas sakız ağac 1 & Atlas mastic tree & Anacardiaceae \\
\hline Pistacia khinjuk Stocks & Tree & Melengiç & & Anacardiaceae \\
\hline Pistacia lentiscus $\mathrm{L}$. & Shrub or Shrub-Tree & Sakız ağacı, Akçakesme, Sakızağacı & Mastic tree & Anacardiaceae \\
\hline Pistacia terebinthus L. & Shrub or Small tree & Çitlenbik, Melengiç & Terebinth, Terebinth Tree & Anacardiaceae \\
\hline Polygala comosa Schkuhr & Shrubby & Sütotu & Tufted milkwort & Polygalaceae \\
\hline Polygala pruinosa Boiss. & Half shrubby & $\begin{array}{l}\text { Anadolu süt otu, Haç çiçeği, Yılan } \\
\text { yoncası }\end{array}$ & Milkwort & Polygalaceae \\
\hline Populus tremula $\mathrm{L}$. & Tree & Titrek kavak & Trembling poplar & Salicaceae \\
\hline Potentilla fruticos $\mathrm{L}$. & Shrub & Parmak otu & & Rosaceae \\
\hline Prasium majus L. & Shrub & & & Labiatae \\
\hline Prosopis farcta (Banks et Sol.) Macbride & Shrub & Çeti, Çedi & & Leguminosae \\
\hline Prunus amygdalus Batsch & Shrub or Tree & Badem ağac1 & & Rosaceae \\
\hline Prunus cerasus $\mathrm{L}$. & Shrub or Small tree & Vişne ağac1 & Cherry & Rosaceae \\
\hline Prunus divaricata Ledeb. & Shrub & Yunus eriği & & Rosaceae \\
\hline Prunus domestica $\mathrm{L}$. & Shrub or Tree & Erik ağac1 & Plum tree & Rosaceae \\
\hline Prunus spinosa L. & Shrub & Çakaleriği, Güvem & Blackthorn & Rosaceae \\
\hline Ptilostemon chamaepeuce (L.) Less. & Shrub & Pembe tüyercik & & Compositae \\
\hline Pyrus elaeagnifolia $\mathrm{L}$. & Tree & Ahlat & Wild pear & Rosaceae \\
\hline Pyrus syriaca Boiss. var. syriaca & Tree & Ahlat, Yabani armut & & Rosaceae \\
\hline Quercus brantii Lindley & Tree & Meşe & & Fagaceae \\
\hline Quercus cerris $\mathrm{L}$. & Shrub or Small tree & Türk meşesi, Saçlı meşe & Turkey oak & Fagaceae \\
\hline Quercus coccifera $\mathrm{L}$. & Shrub or Small tree & Kermes meşesi & Kermes oak & Fagaceae \\
\hline Quercus ilex $\mathrm{L}$. & Tree or Tall shrub & $\begin{array}{l}\text { Çalı meşesi, Karagan, Pırnal } \\
\text { meşesi, Pırnar }\end{array}$ & & Fagaceae \\
\hline $\begin{array}{l}\text { Quercus infectoria Olivier subsp. boissieri } \\
\text { (Reuter) O. Schwarz }\end{array}$ & Tree & Meşe & & Fagaceae \\
\hline Quercus macrolepis Kotschy & Shrub or Tree & Palamut, Pullu meşe & Valonia oak & Fagaceae \\
\hline Quercus petraea (Matt.) Lieb. & Shrub or Small tree & Sapsız meşe & Durmast oak, Sessile oak & Fagaceae \\
\hline Quercus pubescens Willd. & Small tree & Tüylü Meşe & & Fagaceae \\
\hline Quercus robur $\mathrm{L}$. & Shrub or Tree & Saplı meșe, Adi meşe & Common oak, English oak & Fagaceae \\
\hline Quercus trojana P.B. Webb & Tree & Makedonya Meșesi & & Fagaceae \\
\hline Reaumuria alternifolia (Lab.) Britten & Shrub & Kör diken & & Tamaricaceae \\
\hline Rhamnus hirtellus Boiss. (END LR lc) & Shrub & Cehri, Karaçalı & & Rhamnaceae \\
\hline Rhamnus oleoides L. & Shrubby & Kör diken & & Rhamnaceae \\
\hline Rhamnus pallasii Fisch. \& C.A. Mey. & Shrub & Cehri & Buckthorn & Rhamnaceae \\
\hline Rhamnus rhodopeus Velenovsky & Shrub & & & Rhamnaceae \\
\hline Rhamnus saxatilis Jacq. & Shrub & & Rock buckthorn & Rhamnaceae \\
\hline Rhamnus tomentella Benth. & Shrub & & & Rhamnaceae \\
\hline Rhododendron caucasium Pallas & Shrub & Komar, Orman gülü & Rhododendron & Ericaceae \\
\hline Rhododendron luteum Sweet & Shrub & Komar, Orman gülü & Rhododendron & Ericaceae \\
\hline Rhododendron ponticum $\mathrm{L}$. & Shrub or Tree & $\begin{array}{l}\text { Ağu, Komar, Orman gülü, Kafil, } \\
\text { Kaful, Kara ağu, Zelenika }\end{array}$ & Rhododendron & Ericaceae \\
\hline Rhododendron ungernii Trautv. & Shrub or Small tree & Komar, Orman gülü & Rhododendron & Ericaceae \\
\hline Rhus coriaria $\mathrm{L}$. & Shrub & Adi sumak & Sicilian sumac & Anacardiaceae \\
\hline Ribes aureum Prush & Shrub & Frenk üzümü, Sarı bektaşiüzümü & Golden currant & Glossulariaceae \\
\hline Rosa canina $\mathrm{L}$. & Shrub & $\begin{array}{l}\text { Yabani gül, Kuşburnu, İtburnu, } \\
\text { Köpekgülü }\end{array}$ & Dog rose, Wild rose, Heprose & Rosaceae \\
\hline Rosa dumalis Bechst. & Shrub & Yabani gül, Kuşburnu, & Glaucous Dog rose & Rosaceae \\
\hline Rosa hemisphaerica J. Herrm. & Shrub & Sarıçiçekli yaban gülü & Yellow flowers wild rose & Rosaceae \\
\hline Rosa micrantha $\mathrm{Sm}$ & Shrub & Kuşburnu & Smallflower sweetbrier & Rosaceae \\
\hline Rosa montana Chaix & Shrub & Dağ gülü & & Rosaceae \\
\hline
\end{tabular}




\begin{tabular}{|c|c|c|c|c|}
\hline Rosa pulverulenta Bieb. & Shrub & Yabani gül & Wild rose & Rosaceae \\
\hline Rosa sempervirens $\mathrm{L}$. & Shrub & Deli gül, Yabani gül & & Rosaceae \\
\hline Rubus caesius $\mathrm{L}$. & Shrub & & & Rosaceae \\
\hline Rubus canescens DC. & Shrub & Bögürtlen & Blackberry & Rosaceae \\
\hline Rubus discolor Weihe et Nees. & Shrub & Himalaya böğürtleni & $\begin{array}{l}\text { Himalayan blackberry, } \\
\text { Himalayaberry }\end{array}$ & Rosaceae \\
\hline Rubus fruticosus $\mathrm{L}$. & Shrub & Böğürtlen & & Rosaceae \\
\hline Rubus sanctus Schreber & Shrub & $\begin{array}{l}\text { Böğürtlen, Kutsal böğürtlen, Mora } \\
\text { dikeni }\end{array}$ & Blackberry & Rosaceae \\
\hline Ruscus aculeatus L. & Shrub & Tavşan kirazı & Butcher's brome & Rosaceae \\
\hline Ruta chalepensis $\mathrm{L}$. & Half shrubby & Sedef otu, Kokarsedef & & Rutaceae \\
\hline Salix alba $\mathrm{L}$. & Tree & Aksöğüt & White willow & Salicaceae \\
\hline Salix triandra $\mathrm{L}$. & $\begin{array}{c}\text { Shrubby or Shrub- } \\
\text { Tree }\end{array}$ & Badem yapraklı söğüt & Almond willow & Salicaceae \\
\hline Salvia fruticosa Miller (NB VU) & Shrubby & Adaçayı & Triloba sage & Labiatae \\
\hline Salvia pachystachys Trautv. & $\begin{array}{l}\text { Shrubby, Shrub or } \\
\text { Woody-herb }\end{array}$ & Kalın başaklı adaçayı & Rough-spike sage & Labiatae \\
\hline Sarcopoterium spinosum (L.) Spach & Shrub & Aptesbozan & & Rosaceae \\
\hline Satureja juliana $\mathrm{L}$. & Shrubby & Çalımsı geyik otu & Savory, Micromeria & Labiatae \\
\hline Satureja parnassica Heldr.\& Sart. ex Boiss. & Shrubby & Sipil geyik otu & & Labiatae \\
\hline Satureja spicigera (C. Koch) Boiss. & Shrubby & Trabzon kekiği & Savory & Labiatae \\
\hline Satureja thymbra $\mathrm{L}$. & Shrubby & Pembe geyikotu & Pink savory, Barrel sweetener & Labiatae \\
\hline Sorbus aria $($ L.) Crantz & Shrub & Kocakarı yemişi & Common whitebeam & Rosaceae \\
\hline Spartium junceum $\mathrm{L}$. & Shrub & $\begin{array}{l}\text { Katırtırnağı, Saz kaytanotu Borcak, } \\
\text { Borçoh, Boruk, Kuş çubuğu }\end{array}$ & Spanish brom, Weaver's broom & Leguminosae \\
\hline Stachys iberica Bieb. & Half shrubby & Dağ çayı & Woundwort, Lamb's Ear & Labiatae \\
\hline $\begin{array}{l}\text { Stachys iberica Bieb. subsp. stenostachya } \\
\text { (Boiss.) Rech. }\end{array}$ & Half shrubby & & & Labiatae \\
\hline Stachys lavandulifolia Vahl. & Half shrubby & $\begin{array}{l}\text { Mor çiçekli karabaş otu, Eşek otu, } \\
\text { Tokalı çay, Tüylü çay }\end{array}$ & Woundwort, Lamb's Ear & Labiatae \\
\hline Styrax officinalis L. & Shrub or Rarely tree & Ayı findığı, Çakıldak, Tespih ağacı & Styrax tree & Styracaceae \\
\hline Tamarix articulata Vahl. & Shrub or Tree & & \begin{tabular}{|l} 
Athel tamarisk \\
\end{tabular} & Tamaricaceae \\
\hline Tamarix parviflora DC. & Shrub & Ilgin & Tamarisk & Tamaricaceae \\
\hline Tamarix smyrnensis Bunge. & Shrub & Ilgin & Tamarisk & Tamaricaceae \\
\hline Tamarix tetrandra Pallas ex M. Bieb. & Shrub & Ilgin & Tamarisk & Tamaricaceae \\
\hline Tanacetum abrotanifolium (L.) Druce & Shrub or Tree & Ala renkli solucan otu & & Compositae \\
\hline Tanacetum armenum (DC.) Schultz Bip. & Shrub or Tree & Gümüșdüğme & \begin{tabular}{|l|} 
Feverfew, Featherfew, Flirtwort \\
\end{tabular} & Compositae \\
\hline Teucrium chamaedrys $\mathrm{L}$. & Half shrubby & Yer meşesi, Bodur meşe & Germander, Wall germander & Labiatae \\
\hline Teucrium pollium $\mathrm{L}$. & Half shrubby & $\begin{array}{l}\text { Mayasıl otu, Taş kekiği, Acı ot, Ak } \\
\text { sedef otu, Anababa kekiği }\end{array}$ & Hairy germander & Labiatae \\
\hline Thuja orientalis $\mathrm{L}$. & Shrub or Tree & Doğu mazısı & Morpankhi, Thujone & Cupressaceae \\
\hline Thymbra spicata $\mathrm{L}$. & Shrub & Karabaș kekik & Spiked thyme & Labiatae \\
\hline Thymus fallax Fisch. et C.A. Mey. & Shrub & & & Labiatae \\
\hline Thymus kotschyanus Boiss. et Hohen. & Shrub & & & Labiatae \\
\hline Thymus leucotrichus $\mathrm{Hal}$. & Shrub & & & Labiatae \\
\hline Thymus sipyleus Boiss. & Shrubby & Yastıklı kekik & & Labiatae \\
\hline Thymus transcaucasicus Ronniger (NB VU) & Shrub & & & Labiatae \\
\hline Thymus zygioides Griseb. & Shrub & & & Labiatae \\
\hline Ulmus minör Miller & Tree & $\begin{array}{l}\text { Ova karaağacı, Gürgen yapraklı } \\
\text { karaağaç }\end{array}$ & Smooth leaved elm, English elm & Ulmaceae \\
\hline Vaccinium arctostaphylos $\mathrm{L}$. & Shrub & Yaban mersini, Ayı üzümü & Caucasian whortleberry & Ericaceae \\
\hline Veronica multifida L. (END LR lc) & Half shrubby & Yavşan otu, Venüs çiçeği & Speedwell & $\begin{array}{c}\text { Scrophulariacea } \\
e\end{array}$ \\
\hline Vibirnum lantana $\mathrm{L}$. & Shrub & Germișek, Germeșik, Germeșe & Wayfaring tree & Caprifoliaceae \\
\hline Vibirnum opulus L. & $\begin{array}{l}\text { Shrub or Small } \\
\text { shrub-Tree }\end{array}$ & Kartopu, Geleboru, Girabolu & Wayfaring tree & Caprifoliaceae \\
\hline Vincetoxicum hirundinaria Medicus & Shrubby & Panzehir otu, Kırlangıç otu & Swallow wort & Asclepiadaceae \\
\hline Vitex agnus-castus $\mathrm{L}$. & $\begin{array}{l}\text { Shrub or Rarely } \\
\text { small tree }\end{array}$ & Hayıt, İffet ağac1, Rahip biberi & Chaste tree & Verbenaceae \\
\hline Ziziphora clinopodioides Lam. & Half shrubby & Dağ reyhanı, Kır nanesi, Nane ruhu & Persian wild thyme & Labiatae \\
\hline
\end{tabular}

END LR lc: Endemic Plants, NB VU: Non-Endemic Rare Plants

Table 2. Numbers of families, genus and taxa with growth forms and ratios (\%) of some shrub and Tree species found in the grassland-pasture and natural areas of Turkey

\begin{tabular}{|r|l|c|c|c|c|c|}
\hline \multicolumn{1}{|c|}{ Family } & Genus & $\boldsymbol{\%}$ & Taxa & $\boldsymbol{\%}$ & Life Forms \\
\hline 1 & Rosaceae & 14 & 13.0 & 38 & 16.7 & Shrub (21), Tree (10), Shrub or Tree (7) \\
\hline 2 & Leguminosae (Fabaceae) & 12 & 11.1 & 36 & 15.9 & Shrub (36) \\
\hline 3 & Labiatae (Lamiaceae) & 11 & 10.2 & 25 & 11.0 & Shrub (25) \\
\hline 4 & Chenopodiaceae & 7 & 6.48 & 7 & 3.08 & Shrub (6), Shrub or Tree (1) \\
\hline 5 & Ericaceae & 4 & 3.70 & 9 & 3.96 & Shrub (7), Shrub or Tree (2) \\
\hline 6 & Compositae (Asteraceae) & 4 & 3.70 & 6 & 2.64 & Shrub (4), Shrub or Tree (2) \\
\hline 7 & Oleaceae & 4 & 3.70 & 4 & 1.76 & Shrub (2), Tree (1), Shrub or Tree (1) \\
\hline 8 & Anacardiaceae & 3 & 2.78 & 6 & 2.64 & Shrub (2), Tree (1), Shrub or Tree (3) \\
\hline 9 & Tamaricaceae & 3 & 2.78 & 6 & 2.64 & Shrub (5), Shrub or Tree (1) \\
\hline 10 & Cupressaceae & 2 & 1.85 & 9 & 3.96 & Shrub (6), Tree (1), Shrub or Tree (2) \\
\hline 11 & Rhamnaceae & 2 & 1.85 & 7 & 3.08 & Shrub (7) \\
\hline 12 & Cistaceae & 2 & 1.85 & 4 & 1.76 & Shrub (3), Tree (1) \\
\hline 13 & Caprifoliaceae & 2 & 1.85 & 3 & 1.32 & Shrub (2), Shrub or Tree (1) \\
\hline
\end{tabular}




\begin{tabular}{|c|c|c|c|c|c|c|}
\hline 14 & Salicaceae & 2 & 1.85 & 3 & 1.32 & Tree (2), Shrub or Tree (1) \\
\hline 15 & Eleagnaceae & 2 & 1.85 & 2 & 0.88 & Shrub (1), Shrub or Tree (1) \\
\hline 16 & Fagaceae & 1 & 0.93 & 10 & 4.41 & Tree (4), Shrub or Tree (6) \\
\hline 17 & Capparaceae & 1 & 0.93 & 5 & 2.2 & Shrub (5) \\
\hline 18 & Thymelaeaceae & 1 & 0.93 & 5 & 2.2 & Shrub (5) \\
\hline 19 & Berberidaceae & 1 & 0.93 & 4 & 1.76 & Shrub (4) \\
\hline 20 & Plumbaginaceae & 1 & 0.93 & 4 & 1.76 & Shrub (4) \\
\hline 21 & Cannabaceae & 1 & 0.93 & 2 & 0.88 & Tree (2) \\
\hline 22 & Ephedraceae & 1 & 0.93 & 2 & 0.88 & Shrub (2) \\
\hline 23 & Moraceae & 1 & 0.93 & 2 & 0.88 & Shrub or Tree (2) \\
\hline 24 & Pinaceae & 1 & 0.93 & 2 & 0.88 & Tree (2) \\
\hline 25 & Polygalaceae & 1 & 0.93 & 2 & 0.88 & Shrub (2) \\
\hline 26 & Aceraceae & 1 & 0.93 & 1 & 0.44 & Shrub or Tree (1) \\
\hline 27 & Apocynaceae & 1 & 0.93 & 1 & 0.44 & Shrub (1) \\
\hline 28 & Aquifoliaceae & 1 & 0.93 & 1 & 0.44 & Shrub or Tree (1) \\
\hline 29 & Asclepiadaceae & 1 & 0.93 & 1 & 0.44 & Shrub (1) \\
\hline 30 & Betulaceae & 1 & 0.93 & 1 & 0.44 & Tree (1) \\
\hline 31 & Buxaceae & 1 & 0.93 & 1 & 0.44 & Shrub (1) \\
\hline 32 & Cornaceae & 1 & 0.93 & 1 & 0.44 & Tree (1) \\
\hline 33 & Frankeniaceae & 1 & 0.93 & 1 & 0.44 & Shrub (1) \\
\hline 34 & Globulariaceae & 1 & 0.93 & 1 & 0.44 & Shrub (1) \\
\hline 35 & Glossulariaceae & 1 & 0.93 & 1 & 0.44 & Shrub (1) \\
\hline 36 & Guttiferae (Hypericaceae) & 1 & 0.93 & 1 & 0.44 & Shrub (1) \\
\hline 37 & Lauraceae & 1 & 0.93 & 1 & 0.44 & Shrub or Tree (1) \\
\hline 38 & Liliaceae & 1 & 0.93 & 1 & 0.44 & Shrub (1) \\
\hline 39 & Linaceae & 1 & 0.93 & 1 & 0.44 & Shrub (1) \\
\hline 40 & Myrtaceae & 1 & 0.93 & 1 & 0.44 & Shrub (1) \\
\hline 41 & Ranunculaceae & 1 & 0.93 & 1 & 0.44 & Shrub (1) \\
\hline 42 & Rubiaceae & 1 & 0.93 & 1 & 0.44 & Shrub (1) \\
\hline 43 & Rutaceae & 1 & 0.93 & 1 & 0.44 & Shrub (1) \\
\hline 44 & Scrophulariaceae & 1 & 0.93 & 1 & 0.44 & Shrub (1) \\
\hline 45 & Styracaceae & 1 & 0.93 & 1 & 0.44 & Shrub or Tree (1) \\
\hline 46 & Ulmaceae & 1 & 0.93 & 1 & 0.44 & Tree (1) \\
\hline 47 & Umbelliferae & 1 & 0.93 & 1 & 0.44 & Shrub (1) \\
\hline 48 & Verbenaceae & 1 & 0.93 & 1 & 0.44 & Shrub or Tree (1) \\
\hline \multirow[t]{2}{*}{49} & Zygophyllaceae & 1 & 0.93 & 1 & 0.44 & Shrub (1) \\
\hline & Total & 108 & 100 & 227 & 100 & 227 \\
\hline
\end{tabular}

\section{References}

[1] Alan, M., Ekiz, H. (2001). A vegetation survey in forest range of Küredag1-Bala. Tarım Bilimleri Dergisi- J. Agric. Sci., 7(4): 62-69.

[2] Alaturk, F., Alpars, T., Gökkuş, A., Coşkun, E., Akbağ, H.I. (2014). Seasonal Changes in the Nutrient Contents of Some Shrub Species. COMU Journal of Agriculture Faculty, 2(1): 133-141.

[3] Altın, M., A. Gokkus and A. Koc. (2011). Grassland and Pasture Management Volume 2 (Basic Principles). Publication of General Directorate of Agricultural Production and Development, the Turkish Ministry of Agriculture of Rural Affairs, Ankara.

[4] Anonymous, (2017a). National Pasture Usage and Management Project (http://ulusalmeratagem.gov.tr/turler.asp?pg=2\&srcItem=Rosaceae\&srcCol=familya, AD: 14.08.2017).

[5] Anonymous, (2017b). Turkish Crops Data Service (TUBIVES) (http://www.tubives.com /index.php?sayfa=hizli_ara, AD: 21.08.2017).

[6] Anonymous, (2017c). Missouri Botanical Garden. (http://www.missouribotanicalgarden .org/PlantFinder/PlantFinderDetails.aspx?kempercode=f247, AD: 18.08.2017). 
[7] Anonymous, (2017d). United States Department of Agriculture (USDA), Natural Resources Conservation Service (https://plants.usda.gov/core/profile?symbol=RHSA7, AD: 18.08.2017)

[8] Aybeke, M., Kurt, C., Semerci, A. (2007). Edirne İli Çayır Mera Bitkileri. Cilt 1, Baklagiller. Tarım ve Köyişleri Bakanlığı Tarımsal Araştırmalar Genel Müdürlüğü, Trakya Tarımsal Araştırma Enstitüsü Müdürlügü, Edirne.

[9] Babalık, A., Fakir, H. (2007). The Effects of Goat Grazing on Leaf Morphology of Some Shrub Species in Kozağaci Highland of Davraz Mountain (Isparta). Journal of the Faculty of Forestry of Süleyman Demirel University, 2:1-8.

[10] Bakır, Ö. (1985). Çayır ve Mera Islahı (in Turkish). Ankara University Agricultural Faculty, Pub. No: 917, Textbook No: 272, Ankara, Turkey.

[11] Çetiner, M. (2009). Yapay Bir Merada Otlatmanın Bitki Örtüsü ve Toprak Özelliklerine Etkisi. Yüksek Lisans Tezi, Çanakkale Onsekiz Mart Üniversitesi, Fen Bilimleri Enstitüsü, Tarla Bitkileri Bölümü Anabilim Dalı, Çanakkale.

[12] Cetiner, M., Genç, S., Gökkuş, A. (2015). Biga (Çanakkale) İlçesi Gerlengeç Köyü Mera Islahı ve Yönetimi Çalışması. 11. Tarla Bitkileri Kongresi (7-10 Eylül 2015, Çanakkale), Poster Bildiriler, Cilt 2, s. 63-67.

[13] Çınar, S., Hatipoğlu, R., Avcı, M., İnal, İ., Aydemir, S.K., Yücel, H. (2011). Çukurova'nın Geçit Kesimi Meralarında Yabancı Ot Mücadelesi Üzerine Bir Araştırma. IX. Türkiye Tarla Bitkileri kongresi, 12-15 Eylül 2011, Bursa, Cilt III, s. 1674-1679.

[14] Çınar, S., Hatipoğlu, R., Avc1, M., İnal, İ., Yücel, C., Avağ, A. (2014). A research on the vegetation structures of the pastures in district Kirıkhan, Hatay. Journal of Agricultural Faculty of Gaziosmanpasa University, 31(2): 52-60.

[15] Çınar, S., Hatipoğlu, R., Avcı, M. (2015). Effects of Some Weed Control Methods on Hay Yield, Botanical Composition and Forage Quality of a Rangeland in the Mediterranean Region. Journal of Agricultural Sciences, 21: 39-49.

[16] Ekim, T., Koyuncu, M., Vural, M., Duman, H., Aytaç, Z., Adıgüzel, N. (2000). Red Data Book of Turkish Plants (Pterdophyta and Spermatophyta) (in Turkish). Türkiye Tabiatını Koruma Derneği ve Van Yüzüncü Yıl Üniversitesi Yayınları, 246s, Barışcan Ofset, Ankara.

[17] Ertekin, S. (2002). Karacadağ Bitki Çeşitliliği. Sürdürülebilir Kırsal ve Kentsel Kalkınma Derneği Yay. (http://www.surkal.org.tr/dynamiccontent/ 2_karacadagbitkicesitliligi raporu.pdf, Erişim Tarihi: 04.08.2017). 
[18] Genç, S., Gökkuş, A. (2015). Çanakkale İlinde Uygulanan Mera Islahı ve Yönetimi Projelerinin Değerlendirilmesi. 11. Tarla Bitkileri Kongresi (7-10 Eylül 2015, Çanakkale), Poster Bildiriler, Cilt 2, s. 59-62.

[19] Gökkuş, A., Baytekin, H., Hakyemez, B.H., Özer, İ. (2001). Çanakkale’nin Sürülüp Terk Edilen Çalılı Meralarında Yeniden Bitki Gelişimi. Türkiye 4. Tarla Bitkileri Kongresi. Cilt: III, Çayır Mera Yem Bitkileri: 17-21 Eylül, Tekirdağ, s. 13-18.

[20] Gökkuş, A., Parlak, A.Ö., Hakyemez, B.H., Baytekin, H., Parlak, M. (2009). Maki Örtüsünde Yer Alan Bitki Türlerinin Botanik Özellikleri ile Besleme Değerlerindeki Değişimin Belirlenmesi. TÜBİTAK 1060458 No'lu Proje Sonuç Raporu, 148s.

[21] Gökkuş, A., Parlak, A.Ö., Baytekin, H., Hakyemez, B.H. (2013). Change of Mineral Composition of Herbaceous Species at the Mediterranean Shrublands. Journal of Tekirdag Agricultural Faculty, 10(1): $1-10$.

[22] Gökten, A. (1997). Çukurova Bölgesinde Çalı Vejetasyonunun Baskın Olduğu Meralarda Mekanik ve Kimyasal Yöntemlerle Mera Islah Olanakları. Yüksek Lisans Tezi, Çukurova Üniversitesi Fen Bilimleri Enstitüsü Tarla Bitkileri Anabilim Dalı, Adana.

[23] Kokten, K., Hatipoglu, R., Tukel, T. (2003). Çukurova Bölgesi'nde Çalı Vejetasyonunun Baskın Olduğu Meralarda Uygulanan Değişik Mera Islahı Yöntemlerinin Mera Verimi ve Botanik Kompozisyonuna Etkisi. J. Agric. Fac.MKU, 8 (1-2): 33-40.

[24] Öten, M., Kiremitci, S., Erdurmuş, C., Soysal, M., Kabaş, Ö., Avc1, M. (2016). Determination of the Botanical Composition of Some Rangeland in Antalya Province. Atatürk Univ., J. of the Agricultural Faculty, 47 (1): 23-30.

[25] Özen, F., Türk, M. (2014). The effects of the frequency of trees on vegetation in forest gap rangelands. SDU Faculty of Forestry Journal, 15: 9-14.

[26] Sayar, M.S., Anlarsal, A.E., Basbag, M. (2010). Current situation, problems and solutions for cultivation of forage crops in the Southeastern Anatolian Region. J. Agric. Fac. HR.U. 14: 59-67.

[27] Sayar, M.S., Han, Y., Başbağ, M., Gül, İ., Polat, T. (2015). Rangeland Improvement and Management Studies in Southeastern Anatolia Region of Turkey. Pakistan Journal of Agricultural Sciences, 52(1): 9-18.

[28] Serin, Y., Tan, M., Koç, A., Zengin, H., Aksoy, A., Hamzaoğlu, E., Karaca, A., Şentürk, T., Özbay, O. (2008). Türkiye'nin Çayır ve Mera Bitkileri. Tarım ve Köyişleri Bakanlığı Tarımsal Üretim ve Geliştirme Genel Müdürlüğü, Ankara. 
[29] Serin, Y., Zengin, H., Tan, M., Koç, A., Erkovan, İ., Avcıŏlu, R., Soya, H., Geren, H., Gemici, Y., Kendir, H., Sancak, C., Parlak, A.Ö., Öztekin, M., Özbay, O. (2005). Türkiye'nin Çayır ve Mera Bitkileri Kılavuzu. Tarım ve Köyişleri Bakanlığı Tarımsal Üretim ve Geliştirme Genel Müdürlüğü, Ankara.

[30] Seydoşoğlu, S., Saruhan, V. (2015). Diyarbakır İli Bismil İlçesi Taban Meralarının Botanik Kompozisyonlarının Belirlenmesi. 11. Tarla Bitkileri Kongresi (7-10 Eylül 2015, Çanakkale), Poster Bildiriler, Cilt 2, s. 33-38.

[31] Şen, Ç. (2010). Kilis İlinin Bazı Köylerindeki Meralarda Vejetasyon Yapısı Üzerine Bir Araştırma. Yüksek Lisans Tezi, Çukurova Üniversitesi Fen Bilimleri Enstitüsü Tarla Bitkileri Anabilim Dalı, Adana.

[32] Tashev, A. (2013). Pulsatilla Styriaca (Pritzel) Simonk. (Ranunculaceae) - A New Species for Bulgarian Flora. Bulgarian Journal of Agricultural Science, 19 (2): 347-352.

[33] Temel, S., Tan, M. (2013). The growth patterns of shrub species in maquis in Mediterranean region. Iğdir University Journal of the Institute of Science and Technology, 3(2), 77-86.

[34] Temel, S., Kır A.E. (2015). Determination of grazing preferences of the small ruminants based on seasonal periods of some shrub and tree species. International Journal of Agriculture and Wildlife Science, 1(1), 31-39.

[35] Tolunay, A., Ayhan, V., Adıyaman, E., Akyol, A., İnce, D. (2009). Dry Matter Yield and Grazing Capacity of Kermes Oak (Quercus coccifera L.) Shrubland for Püre Hair Goat (Capra hircus L.) Breeding in Turkey's Western Mediterranean Region. Journal of Animal and Veterinary Advances, 8(2): 368-372.

[36] Tükel, T., Hatipoğlu, R. (1997). Çayır Mera Amenajmanı. Çukurova Üniversitesi Ziraat Fakültesi Genel Yayın No: 191. Ders kitaplan Yayın No: A-59, Adana.

[37] Türkmen, C., Müftüoğlu, N.M., Kavdır, Y. (2013). Change of some soil quality characteristics under different pasture reclamation methods of rangelands. Tartm Bilimleri Dergisi, -J. Agric. Sci., 19: 245-255.

[38] Uslu, Ö.S. (2005). Kahramanmaraş İli Türkoğlu İlçesi Araplar Köyü Yeniyapan Merasında Botanik Kompozisyonun Tespiti ve Farklı Gübre Uygulamalarının Meranın Verim ve Botanik Kompozisyonuna Etkileri Üzerinde Araştırmalar. Doktora Tezi, Çukurova Üniversitesi Fen Bilimleri Enstitüsü Tarla Bitkileri Anabilim Dalı, Adana.

[39] Uysal, A., Bilgen, M., Özyiğit, Y. (2015). Çalı Meralarında Gençleştirme İşleminin Bitki Gelişimine Etkisi. 11. Tarla Bitkileri Kongresi (7-10 Eylül 2015, Çanakkale), Poster Bildiriler, Cilt 2, s. 55-58. 
Middle East Journal of Science (2017) 3(2): 115-128

[40] Uzun, F., Garipoğlu, A.V., Dönmez, H.B. (2015). Using Goats for Weeds Control in Pastures. International Journal of Agriculture and Wildlife Science (IJAWS), 1(1): 40-50.

[41] Yavuz, T., Sürmen, M., Sürmen, B., Töngel, Ö., Kutbay, H.G., Yilmaz, H. (2013). Evaluation of Cuminum cyminum L. Invasiveness Potential in Grassland and Pastures of Central Black Sea Region (Samsun/TURKEY). Journal of Anatolian Natural Sciences, 4(2): 28-32.

[42] Yılmaz, K.T. (1996). Akdeniz Doğal Bitki Örtüsü. Çukurova Üni. Ziraat Fak. Genel Yayın No: 141. 